\title{
Estigma del sistema de género: aprendizaje de los modelos normativos, bullying y estrategias de resiliencia
}

\author{
Susana BARÓN VIOQUE \\ Facultad de Psicología, Universidad Complutense de Madrid \\ susana_baron@psi.ucm.es \\ Michele CASCONE \\ Facultad de de Psicología, Universidad Complutense de Madrid \\ michelecascone@pdi.ucm.es \\ Carlos MARTínez VALLE \\ Facultad de Educación, Universidad Complutense de Madrid \\ carlos03@ucm.es
}

Recibido: 06-05-2013

Aceptado: 26-11-2013

\begin{abstract}
Resumen
El presente artículo repasa de manera crítica el estado de la cuestión de la creación, transmisiónaprendizaje y manejo-afrontamiento del estigma y la discriminación contra las mujeres y las personas LGTBQ. Tras seguir, en el marco de la psicología social, la historia y usos del concepto de estigma y considerar las teorías del heterosexismo y genderismo, proponemos el concepto de estigma de género como término inclusivo de todos los procesos de estigmatización que afectan tanto a las mujeres como a quienes no se ajustan al modelo heteronormativo y, por tanto, tienen su origen en el sistema de género. Dentro de la psicología básica, nos detendremos en el recientemente desarrollado modelo del condicionamiento evaluativo por considerarlo el más apropiado para el estudio de la transmisiónaprendizaje del estigma de género, pues se centra en el estudio del aprendizaje del gusto. Seguiremos repasando el bullying de género como instrumento de aprendizaje y efecto del estigma. Finalmente, tras apuntar las consecuencias psico-sociales del estigma en sus portadores, atenderemos a las estrategias que estos desarrollan para manejar y enfrentarse a él. Para ello, seguiremos las novedosas propuestas de la "psicología positiva", en particular profundizamos en los conceptos de empoderamiento y resiliencia.
\end{abstract}

Palabras clave: estigma, resiliencia, estigma de género, heteronormatividad, heteroseximo, bullying 


\title{
Gender Stigma: Learning of Normative models, Bullying and, Resilience Strategies
}

\begin{abstract}
This article proposes a critical review of the literature about the creation, transmission-learning and dealing with stigma and discrimination of both women and LGTBQ people. The article reviews the evolution of the uses of the concept of stigma in the social psychology and, considering the Heterosexism and Genderism theories, proposes the concept of "gender stigma" for a comprehensive study of the stigmatization process that originate in the gender system and affect women and those that doesn't fit into the heteronormative frames. Considering the different transmission-learning models proposed by basic psychology, the article focuses on the evaluative conditioning, as this new approach focuses on (dis)liking which is at the center of transmission and learning of a stigma. The article focuses on gender bullying as an instrument of transmission and learning of the stigma and its effect. After analyzing the psycho-social consequences of gender stigma for its bearers, the article considers, in the perspective of "positive psychology", the coping and empowerment strategies used by stigmatized people to deal with stigma and the creation of resilience.
\end{abstract}

Key words: Stigma, Heterosexism, Genderism, Gender Stigma, Heteronormativity, Resilience, Bullying.

\section{Referencia normalizada}

Barón Vioque, S.; Cascone, M.; Martínez Valle, C. (2013). "Estigma del sistema de género: aprendizaje de los modelos normativos, bullying y estrategias de resiliencia”. Política y Sociedad, Vol.50 Núm. 3 837-864

Sumario: Introducción 1. El proceso de estigmatización 2. De la heteronormatividad al estigma de género 3. Cómo aprendemos el estigma: mecanismos básicos de aprendizaje y transmisión 4. El Bullying o acoso de género 5. Estigma: efectos y estrategias de enfrentamiento 6. Consideraciones finales. Bibliografía.

\section{Introducción}

La pervivencia de la discriminación de la mujer y las minorías sexuales más de 200 años después de la publicación de la Déclaration des Droits de la Femme et de la Citoyenne de Olympe de Gouges y medio siglo después de Stonewall, por poner dos hitos convencionales, de un lado, y también los éxitos alcanzados por los movimientos feministas y LGTB ${ }^{1}$ del otro, exigen, tanto re-pensar las bases psicosocia-

${ }^{1}$ LGTB: Acrónimo que se refiere a Lesbianas, Gays, Transexuales y Bisexuales, también reconocido en el contexto del estado español en su versión más extensa de LGTBQI, que añade los sujetos Queer/Questioning y las personas Intersexuales. 
les sobre las que se asienta esa discriminación, como considerar las respuestas de los estigmatizados que han permitido su avance social. El presente artículo propone un acercamiento crítico al estado de la cuestión de algunos problemas y conceptos abordados por el Proyecto Hermes, un proyecto europeo que vincula la investigación y la intervención de/en las distintas formas de discriminación relacionadas con el sistema de género ${ }^{2}$.

Para seguir la secuencia de la creación social, la transmisión-aprendizaje y el afrontamiento del estigma, el presente artículo revisa la literatura de la psicología social, básica y la así denominada "psicología positiva” sobre, respectivamente, los procesos de creación-atribución del estigma a mujeres y personas LGTB, la transmisión-aprendizaje de ese estigma y la resistencia a los efectos de la discriminación y sugiere líneas futuras de investigación para profundizar en el conocimiento y la reacción a la discriminación por razones de género. Siguiendo la evolución conceptual del término estigma, con el que la psicología social ha estudiado la discriminación de las diferencias sexuales y, a través del análisis de las teorías del heterosexismo y genderismo, el artículo acuña y propone un nuevo concepto, el estigma de género, como término inclusivo para designar los procesos de estigmatización que tienen su origen en el sistema ideológico patriarcal y que afectan tanto a las mujeres como a las personas que no se ajustan al modelo heteronormativo. Un concepto que, debidamente operacionalizado, podría servir para guiar ulteriores investigaciones empíricas en torno a la discriminación conjunta de mujeres y personas LGTB. De igual manera, de entre los modelos con los que la psicología básica estudia la transmisión y aprendizaje, propondremos el del condicionamiento evaluativo como un posible y poco explorado modelo para la comprensión de la transmisión y aprendizaje del estigma de género. Explora el bullying de género como efecto e instrumento de transmisión-aprendizaje del estigma. Finalmente, el artículo revisa el estado de la investigación sobre las estrategias de los estigmatizados para afrontar la estigmatización, fijando la atención en los conceptos de empoderamiento y resiliencia, conceptos todavía no suficientemente estudiados en las personas que han sufrido la estigmatización de género.

\section{El proceso de estigmatización}

La psicología social, que, a través del enfoque social-cognitivo, es una de las disciplinas que más se ha interesado por entender cómo las personas construyen categorías sociales y las vinculan a creencias estereotipadas dando lugar a la discriminación (Crocker et al, 1998), ha desarrollado, entre otras, dos líneas teóricas para tratar estos procesos, la del "prejuicio" de la escuela de Allport (1954) que estudia

${ }^{2}$ El lector encontrará más información sobre el proyecto Hermes en artículo introductorio a este número de la revista. 
la creación de categorías diferenciadas, el prejuicio, la discriminación y la violencia social; y, la del "estigma” de Goffman (1963). Intentaremos aquí recoger las aportaciones teóricas más relevantes al concepto de "estigma”, elegido por el grupo de investigación Hermes como punto de partida para abordar la investigación e intervención en episodios de violencia y discriminación contra las personas que no se ajustan al modelo heteronormativo.

Encontramos los primeros intentos sistemáticos de definición del "estigma” en la obra de Goffman (1963), de la que parten las reflexiones ulteriores que perfilan el concepto según sus enfoques de referencia y los ámbitos disciplinarios en los que se ha empleado. Para el autor canadiense, el estigma se desarrolla en las interacciones sociales, cuando la identidad social actual de un individuo -sus atributos- no satisface las expectativas sociales. Goffman define el estigma como "un atributo profundamente desacreditador dentro de una interacción social particular", que reduce a su portador, simbólicamente, "de una persona completa y normal a una cuestionada y disminuida en su valor social" (1963: 3 ).

Jones et al. (1984) amplían la concepción de Goffman del estigma como una relación entre un atributo y un estereotipo (1963: 4), para definirlo como una marca, en sentido físico o figurado, que vincula un individuo a una serie de características indeseables (estereotipos). Asimismo, partiendo de la observación de Goffman de que su concepto trata "de relaciones y no atributos" (1963: 3), Sayce (1998) ha vinculado el estigma a otros conceptos, en particular al de discriminación, subrayando que el estigma no es algo "dentro de la persona" sino que se trata de una asignación o etiqueta que otros, dentro de un sistema de relaciones de poder, imponen a una persona o a un grupo. De esta forma, tenemos sujetos activos que rechazan y excluyen a otros, quienes acaban convirtiéndose, a su pesar, en receptores y destinatarios de susodichas actitudes.

Por lo tanto, si para Goffman el estigma es principalmente un atributo, aún no perteneciente a la persona sino atribuido socialmente, gran parte de los autores posteriores lo consideran un proceso social. En particular, Link y Phelan (2001) lo definen como la convergencia o concurrencia interrelacionada de diversos elementos: etiquetado, estereotipado, pérdida de estatus y discriminación; todo ello, insertado en una situación donde se ejerce alguna forma de poder, porque el poder es lo que permite a éstos elementos del proceso de estigmatización tomar forma. Según estos autores, la estigmatización comienza cuando grupos con poder social, económico y/o político reconocen ciertos rasgos diferenciadores y les asignan un valor negativo que se extiende a toda la persona estigmatizada -el efecto halo-, consiguiendo su etiquetamiento social a partir de estas diferencias. Este etiquetado lleva a clasificaciones en categorías separadas, que distinguen el "nosotros" de "ellos"

${ }^{3}$ La creación de límites psicológicos y simbólicos entre los individuos resulta ser un elemento básico constituyente de la identidad, tiende a reflejarse en la constitución de grupos sociales, donde un "nosotros” se contrapone a un "ellos”. Esta contraposición dialéc- 
La estigmatización culmina cuando esta categorización desencadena distintas formas de desaprobación, rechazo, exclusión y discriminación. En estos casos, las personas etiquetadas experimentan una pérdida de estatus social, que reducirá su acceso a cuotas de poder económico, político y social, afectando a su bienestar psicológico, sus posibilidades de empleo y su vida en general.

Entender el estigma como proceso, y no como atributo, permite ver con claridad cómo el atributo que determina una identidad menospreciada por la sociedad es, en realidad, fruto de un acuerdo social en un contexto particular, y que se requieren desequilibrios significativos de poder para originar el estigma. Además, muestra que la estigmatización es una herramienta de poder para mantener las desigualdades sociales y los privilegios de los estigmatizadores. ${ }^{4}$

Ha sido el psicólogo social Herek quien ha vinculado de forma expresa el concepto de estigma con los fenómenos de violencia y discriminación contra las sexualidades no normativas. Su obra ha sido determinante para que el Grupo Hermes eligiese el concepto de estigma frente a otros conceptos y líneas teóricas de la psicología social. Aunque Herek (1984; 2004) reconoce la gran importancia histórico-política del más difundido término homofobia, considera sus limitaciones e imprecisiones. El término homofobia cobró gran importancia a partir de su primer uso en la obra clásica de Weinberg (1972), pues no solo permitió dar un nombre a las vivencias de discriminación, rechazo e invisibilidad que homosexuales y lesbianas sufrían. El término y el discurso en el que se insertaba se convirtieron en instrumentos de lucha que desplazaron el foco de atención. El "problema" de la homosexualidad ya no se encontraba en las personas homosexuales, sino en los intolerantes que les rechazaban. Además, el paradigma de la "homofobia" sirvió como modelo para conceptualizar una gran variedad de actitudes negativas basadas

tica entre nosotros/ellos a nivel grupal, y entre yo/otro a nivel individual, es la base del proceso de formación de la identidad. Pensamos que la identidad es un proceso relacional, una incesante dialéctica entre un Yo en construcción y el Otro, la diferencia, a través de la cual el Yo se va definiendo (Hall, 2003; Bauman, 2005). La identidad, por lo tanto, nunca está determinada en sí misma, "sólo puede construirse a través de la relación con el Otro, la relación con lo que él no es, con lo que justamente le falta, con lo que se ha denominado su afuera constitutivo" (Hall, 2003: 18). Dentro de este juego dialéctico, todas las identidades actúan por medio de la exclusión, a través de la construcción discursiva de un afuera constitutivo y la producción de sujetos abyectos y marginados (Butler, 1990; 1993).

${ }^{4}$ Cualquiera podría desencadenar el proceso de estigmatización (etiquetar un grupo con unos atributos y vincularlos a características negativas hasta desencadenar el rechazo y la discriminación), pero el proceso no se completa si la persona en cuestión no posee el suficiente poder social, económico y político para que sus representaciones tengan consecuencias discriminatorias. Por ejemplo, si consideramos sólo los elementos cognitivos del etiquetamiento y estereotipación, grupos como los abogados, los políticos y las personas blancas podrían ser considerados grupos estigmatizados: son los elementos de pérdida de estatus, relación de poder y discriminación que distingue la estigmatización. 
en el género y en la sexualidad ${ }^{5}$. Empero, tras tres décadas en las que el discurso de la homofobia ha contribuido notablemente al debate público con la visibilidad de la estigmatización y la transformación de la percepción del colectivo de gays y lesbianas, algunas voces han comenzado a revisarlo. Según Herek (1984; 2004), las principales limitaciones del concepto homofobia conciernen a la falta de correspondencia entre la homofobia y las fobias clínicas ${ }^{6}$ y su enfoque centrado sólo en el individuo desde la perspectiva clínica, lo que preteriría u obviaría las causas socioculturales.

Enlazando con las teorías de Goffman, Herek propone el concepto de estigma sexual, basándose en la observación de que la sexualidad y las distintas formas que adopta, están en buena medida socialmente construidas y definidas de una forma jerárquica que revelan desigualdades de poder y status. En concreto, el estigma sexual sería una forma de conocimiento compartido, basado en las ideologías socioculturales que definen la sexualidad, que atribuye un valor negativo a todo comportamiento, identidad, relación, individuo y comunidad no heterosexual, colocándolos en un estatus inferior a los heterosexuales.

Herek observa que, desde el nacimiento, las personas están en contacto con las ideologías y normas socio-culturales de la sexualidad y, a través de sus acciones, éstas eligen expresarlas, reafirmarlas o desafiarlas. De esta manera, el estigma sexual, a nivel individual, puede manifestarse en tres formas distintas:

1) El Enacted sexual stigma, expresado a través del rechazo, ostracismo, los insultos, la abierta discriminación y la violencia. Visto que cualquiera, independientemente de su orientación y prácticas sexuales, puede ser percibido como gay, lesbiana o bisexual, tanto los heteros como los no-heteros pueden ser el blanco del enacted estigma. Además, miembros de ambos grupos pueden perpetrar el estigma.

2) El Felt stigma, que empuja a las personas hetero y no-hetero a utilizar estrategias de presentación de sí mismos para evitar ser percibidos y etiquetados como pertenecientes a una minoría sexual. Las personas hetero y no-hetero pueden controlar sus actitudes, por ejemplo evitando las manifestaciones de afecto hacia personas del mismo sexo o incluso estigmatizando a los demás.

\footnotetext{
${ }^{5}$ Lesbofobia (Kitzinger, 1986); bifobia (Ochs and Deihl, 1992); transfobia (Norton, 1997); effeminofobia (Sedgwick, 1993).

${ }^{6}$ El principal componente emocional de una fobia es la ansiedad, mientras que en la homofobia es el odio (Haaga, 1991; Herek and Berril, 1992). Además, el individuo que sufre la fobia considera sus miedos excesivos e irracionales, mientras los homófobos consideran su odio justificado. La conducta disfuncional asociada a las fobias es la evitación, la homofobia conduce eventualente a la agresión. En fin, los sujetos afectados por una fobia desean modificar su condición, mientras el impulso para cambiar la conducta homofóbica ha de venir, usualmente, desde el exterior.
} 
3) El Internalized sexual stigma, un concepto validado experimentalmente, entre otras, por la escala IHP (Herek y Glunt, 1995). Designa la aceptación por heterosexuales o miembros de una minoría sexual del estigma sexual como parte del propio sistema de valores. Para los heterosexuales, el estigma interiorizado se manifiesta a través de actitudes negativas contra las minorías sexuales. Para las minorías sexuales, el estigma interiorizado puede ser dirigido tanto hacia el interior, en forma de self-directed prejudice, (o, con un término más extendido, homofobia interiorizada), como hacia el exterior (los miembros de las minorías sexuales interiorizan los principios del estigma y los aplican a los demás, llegando incluso a adoptar actitudes negativas contra su propio colectivo) (Herek, Cogan, Gillis y Glunt, 1998).

\section{Del estigma sexual al estigma de género}

El concepto de Herek, sin embargo tiene, a nuestro parecer, algunos problemas, comenzando porque puede entenderse de manera naturalizada como relacionado a un invariante biológico, la sexualidad, y también por su inexactitud, pues el estigma no se asigna necesariamente por preferencias o prácticas sexuales. De hecho, la norma que subyace a la adscripción, clasificación, minusvaloración y discriminación del estigma no es el mero hecho sexual. Así, parte de la transmisiónaprendizaje y práctica de la estigmatización se realiza durante la infancia y la pubertad, un periodo en el que las personas no suelen tener un deseo sexual plenamente definido, ni prácticas sexuales con otras personas (Plummer, 2001). Además el bullying de género no se dirige sólo a aquellos niños y jóvenes que pueden ser estigmatizados por la discrepancia de su aspecto y conducta frente a las normas de género, sino también a aquellos que tienen algún pariente o amigo que las quiebra (Clarke et al. 2004) o a aquellos que no están seguros de su orientación (Espelage et al. 2008). Como instrumento de poder, el estigma ha de basarse en sistemas ideológicos y normativos que regulen características más visibles y abarcantes que el propio gusto y la práctica sexual. En este apartado intentaremos profundizar en el origen y naturaleza de estos sistemas ideológicos para proponer un concepto alternativo.

Se ha definido la heteronormatividad (Warner, 1991: 4) como el conjunto de ideologías y normas socio-culturales que sirven para construir una norma sexual a través de la determinación de formas de sexualidad idealizadas y denigradas. A su vez, la heteronormatividad se concretaría principalmente en dos ideologías, el heterosexismo y el genderismo.

El genderismo es la creencia compartida de que sólo hay y, contradictoriamente, sólo debe haber dos géneros, y únicamente estos dos son naturales (Cope y Darke, 1999; Izzo, 2007). El genderismo insiste en que la identidad de género de una persona debe coincidir con su sexo biológico, en que existen conceptos estrictamente definidos de masculinidad y feminidad y, por tanto, comportamientos esperados diferenciados de hombres y mujeres. El genderismo determina la existencia de 
reglas sobre cómo los hombres y las mujeres deben aparentar y actuar en sociedad y cuya quiebra conlleva rechazo y discriminación. Un caso evidente es el de los transexuales, pues la ambigüedad en sus rasgos físicos provoca incomodidad en muchas personas.

Por otro lado, el heterosexismo (Barret y Logan, 2002; Herek, 1986, 1996) hace referencia a la asunción de que todas las personas son heterosexuales y que la heterosexualidad es más deseable que cualquier otra opción sexual. El término heterosexismo indica también la estigmatización, denigración o negación de cualquier opción no heterosexual y esta actitud se emplea para justificar el maltrato, la discriminación y el abuso de personas que no se ajustan a la opción heterosexual. Como hemos destacado anteriormente, según Herek (1996) el heterosexismo sería el medio socio-cultural que da origen y permite perpetuar el estigma sexual.

Las ideologías genderistas y heterosexistas tendrían su origen en la opresión del conjunto de la sociedad por el patriarcado (Butler, 1993; Chodorow, 199; Irigaray, 1977; Pharr, 1988) para mantener el orden establecido, un orden donde un género, el masculino, puede dominar al otro, el femenino. Ambas serían inherentes a una visión naturalizada y dicotómica de los sexos, de los géneros y de las sexualidades. Mantener la creencia de que sólo existen y tienen que existir dos géneros bien diferenciados y que estos dos géneros deben complementarse a través de una unión heterosexual, sustenta la idea de que existen y deben existir diferencias entre ellos, justificando la subordinación psicológica, social, cultural y política de todos los individuos al género masculino, que siempre es mejor valorado en la jerarquía social por su poder y estatus.

Teniendo en cuenta las precedentes aportaciones teóricas, desde el Proyecto Hermes queremos proponer el concepto de estigma de género para incluir todos los procesos de estigmatización que tienen su origen en el sistema ideológico heteronormativo. De esta forma, el concepto de estigma de género englobaría y ampliaría el concepto de estigma sexual y permitiría vislumbrar un origen común a los procesos de estigmatización que afectan tanto a las mujeres (a las que se asigna un rol y status socio-político inferior al de los hombres) como a las personas que no se ajustan a los modelos de género (incluyendo a las personas que performan ${ }^{7} \mathrm{su}$ género fuera de los modelos tradicionales, gays, lesbianas, bisexuales o transexuales). La existencia de un estigma de género se puede inferir de la valoracion social del rol pasivo frente al activo en las relaciones homosexuales, más denigrado, incluso en el mundo homosexual, al ser considerado una "feminizacion" del varón. Suscribimos la hipótesis de Lingiardi y Luci (2006) que asignan un rol central en el origen de la homofobia a la devaluación del afeminamiento, evidenciando de esta

${ }^{7}$ La idea de performatividad (Butler, 1990) presupone que el sujeto construye la realidad y su propia identidad mediante los actos que ejecuta o interpreta. Aplicando esta idea al género, se deriva que el género es, según Butler, substancialmente, aprendido, construido y representado. 
forma un fuerte vínculo entre homofobia y machismo y revelando una conexión entre homofobia y misoginia.

En conclusión, cuando empleamos la palabra estigma de género estamos pensando en un proceso comunicativo-performativo que persigue el control social a través de la imposición habitual de una cosmovisión heteronormativa naturalizada. La imposición de esta norma -el heterosexismo y el genderismo-, se produciría a través de la evaluación de la adecuación a la norma dominante y tendría aspectos de refuerzo hacia aquellos que se adecuan a la norma establecida (mujeres muy femeninas y hombres muy masculinos) y de desprecio/rechazo/violencia contra quienes se alejan de la norma o rompen con ella (hombres amanerados, mujeres masculinas, homosexuales, lesbianas o sujetos transexuales o queer). El grado de violencia que se ejerce dependerá de la divergencia frente a la norma, la radicalidad del cuestionamiento de ésta, la posición del sujeto en el grupo o el rigor en la consideración como norma del aspecto contravenido, entre otros. Consideramos la estigmatización, pues, como un proceso de violencia, en buena parte simbólica, pero que tiene un último refrendo y "validez" en la violencia física. Esto es, contiene siempre la promesa del recurso a la violencia como ultima ratio en el restablecimiento de la norma y la supresión de la diferencia. La estigmatización no se traduce solo en rechazo y exclusión de los grupos de pares o de otros círculos sociales o en injurias frente al diferente, sino que modifica las actitudes y prácticas de todos los envueltos.

\section{Cómo aprendemos el estigma: mecanismos básicos de aprendizaje y trans- misión de la heteronormatividad}

Tras estudiar, desde la perspectiva psicosocial, la creación y adscripción o asignación del estigma de género, precisamos considerar con mayor detalle la transmisión-aprendizaje de los valores de la heteronormatividad, los roles producidos por el estigma, y los hábitos inducidos por ellos. Un ámbito que ha sido abordado desde la psicología básica dedicada al estudio de los procesos básicos de transmisiónaprendizaje, que aplicamos aquí al estigma de género.

Un problema relacionado con la pregunta sobre cómo se trasmite y se perpetúa el estigma, y que ha de ser explicado al mismo tiempo, es el de la variabilidad en las conductas y respuestas a las normas de género, pues éstas pueden variar desde la interiorización, o la modificación hasta el rechazo. La explicación de la variabilidad de la respuesta no es sencilla, porque hay multitud de variables biopsicosociales que interactúan en la determinación de las conductas y no hay un solo mecanismo de adquisición o trasmisión del estigma.

La psicología básica ha perfilado distintos modelos de aprendizaje que parten de una concepción activa del individuo quién, consciente o inconscientemente, de forma controlada o no, modifica, interioriza, reelabora las normas y la cultura en la que está inserto (Seger, 1998). Tanto el condicionamiento clásico como el operante o el aprendizaje vicario pueden dar cuenta de gran parte de las respuestas de ansiedad, incluidos los trastornos, derivados de la convivencia con el estigma de género. 
Con ellos se podrían explicar tanto el rechazo del estigma como sus distintas formas propuestas por Herek, mencionadas en el anterior epígrafe (Enacted, Felt o Internalized estigma). Por ejemplo, en el caso del adolescente enamorado de un compañero de su mismo sexo que es vejado por romper las normas de género, los estímulos incondicionados del amor y el miedo provocarán respuestas incondicionadas contradictorias y concurrentes, conformando un caso complejo de condicionamiento en el que el papel del individuo y sus circunstancias determinará la respuesta prevalente, del rechazo del estigma a su enactment o interiorización.

Frente a estos modelos, en las últimas décadas la literatura ha perfilado lo que se denomina como aprendizaje o condicionamiento evaluativo (De Houwers et al., 2001), sobre el cambio en la valencia hedónica de un estímulo condicionado cuando aparece emparejado a un estímulo incondicionado, impregnado de una valoración hedónica relevante, modificando dicha valencia hedónica del estímulo condicionado en la dirección de la que posee el incondicionado. Este modelo presenta dos ventajas frente a los anteriormente considerados: 1) permitiría explicar de manera más clara la variabilidad de los resultados a los que dan lugar los contradictorios estímulos (con sus correspondientes valencias hedónicas) implicados en la definición de la posición personal frente al estigma, desde el rechazo a la internalización; y 2) tendría mayor capacidad explicativa de los procesos de aprendizaje y transmisión del estigma al centrar su atención justamente en los componentes evaluativos, i.e. en los gustos y preferencias que conforman o impregnan los procesos y los resultados del aprendizaje, en nuestro caso, el componente evaluativo negativo que es un aspecto clave del estigma de género. Ello permitiría explicar ajustadamente la adquisición, el mantenimiento del estigma o la homofobia interiorizada; incluso al margen de lo que en términos dinámicos denominaríamos procesos conscientes.

El condicionamiento evaluativo no es solo un modelo sino también un programa de investigación, pues propone que "la investigación futura debe adoptar un enfoque meta-condicional que no sólo se centre en si una condición determinada es crucial para obtener el condicionamiento evaluativo, sino que examine también cuándo esa condición es crucial” (De Houwers, 2007: 230). Este modelo de aprendizaje no sólo se ha usado para el tratamiento de problemas clínicos como las fobias (Merckelbach et al., 1993) o el estudio de las trasgresiones morales (Bieke, 2011), sino que ha sido aplicado al cambio de actitudes en general (Walter, 2002) y, en particular, a problemas sociales como la reducción de prejuicios raciales (Olson y Fazio, 2006), sexistas y la homofobia (Nyathi, 2008). Estamos aquí lejos de proponer programas muy cuestionables de reeducación a través de experiencias con distinta valencia hedónica, al modo de la naranja mecánica, pero quizás el modelo podría ayudar a conformar espacios en los que la diversidad y la libre elección no se vean coartadas por "condiciones cruciales" contrarias a los individuos. 


\section{El bullying o acoso escolar de género}

El bullying o acoso escolar por razón de género es, al mismo tiempo, el efecto de la estigmatizacion y uno de los principales instrumentos de transmisión y aprendizaje del estigma de género. No es extraño, por tanto, que en los estudios internacionales sobre su prevalencia se configure como un fenómeno endémico en los contextos escolares. En España, el bullying no se ha venido midiendo de manera específica en los grandes estudios de acoso escolar, del Defensor del Pueblo o del Centro de Estudios Rreina Sofia, pero sí contamos con estudios parciales como los de Generelo y Pichardo $(2005,2007)$ con alumnos de Educación Secundaria de la Comunidad de Madrid, y los posteriores, realizados entre otros por FELGTB -COGAM con participación de estos autores. En los primeros, se afirma que alrededor de 1/3 de la muestra de jóvenes insulta, cerca de 1/6 lanza objetos, golpea o aisla y un $3 \%$ ha participado en palizas; Un porcentaje de $13 \%$ de la muestra justifica el trato desigual, un $65 \%$ de los chicos homosexuales fue insultado y un $10 \%$ sufrieron palizas. De hecho, que el acoso tiene carácter de género se ratifica en el hecho de que el 15\% y el $10 \%$ de los chicos que se describieron como heterosexuales también sufrieron, respectivamente, insultos y golpes. En un estudio realizado en el Reino Unido (Mason y Palmer, 1996) se encontró que el 48\% de los estudiantes había experimentado formas de abuso por otros alumnos. En Italia, el estudio de Prati et. al. (2010) sobre una muestra de 863 estudiantes obtiene que casi la mitad de ellos han utilizado insultos relacionados con el género contra compañeros a los que consideran gays y casi $1 / 4$ de la misma los ha proferido contra compañeras a las que consideran lesbianas. Según el autor, el 19,93\% de la muestra puede considerarse abusadores según el criterio de Fonzi (1997) y Olweus (1993) y el 3,71\% ha sufrido actos de bullismo por género con una cadencia al menos semanal.

Como el estigma, el bullying de género puede manifestarse de distintas maneras, desde el insulto y desprecio al aislamiento o distintos grados de violencia física (Rivers, 1996; Warwick et al, 2004). La literatura del bullying (Bacchini, 2000; Fonzi, 1997; Olweus, 1993; Smith et al. 1999) ha identificado tres rasgos esenciales compartidos en todas sus expresiones: (a) la conducta agresiva y la intención de causar daño; (b) la reiteración en el tiempo, y (c) la ocurrencia en relaciones interpersonales y grupales en las que hay diferencia de poder. En particular, la asimetría del poder se basa, en el bullying por género, en la asunción de la superior fortaleza de la virilidad, de la normalidad y del grupo dominante sobre los acosados (Salmivalli et al, 1996).

Numerosos estudios muestran los efectos del acoso por género en la salud mental (Espelage, 2008), pues los abusos se conforman como estresantes que generan a corto y largo plazo abandono escolar, auto-aislamiento, alteraciones en los afectos y las relaciones, depresión, ansiedad, insomnio y conductas autodestructivas que pueden llegar al suicidio. El acoso por género se conforma como un predictor fuerte de problemas sicológicos y riesgos psicopatológicos (Rivers 2004). Esta es la razón por la que la literatura se ha preocupado por los medios para contenerlo o impedirlo, dando relevancia a dos elementos: el ambiente escolar y los grupos de pares. En 
cuanto al ambiente escolar se ha observado que la exposición a un ambiente escolar estigmatizante incrementa el prejuicio contra los homosexuales, especialmente en estudiantes masculinos. Por otra parte, la exposición repetida a episodios de acoso incrementa la propensión a intervenir a favor de la víctima (Prati et al., 2009). Además, el clima escolar percibido por los estudiantes LGTB puede influenciar su adaptación escolar y se ha confirmado que el apoyo de algunos profesores y compañeros puede tener efectos positivos si se da en un ambiente escolar de condena del acoso (Barón y Lacalle, 2011; Murdock y Bolch, 2005).

Mucho más que individuos aislados, los agentes principales del acoso son los grupos de pares, que influencian a lo largo del tiempo a todos los miembros del grupo (Poteat, 2007; Poteat et al., 2007), siendo especialmente propensos al acoso aquellos grupos que tienen una orientacion social dominante y se constituyen en torno a jerarquías que justifican la existencia de grupos dominantes y dominados (Sidanius y Pratto, 1999).

\section{Estigma: efectos y estrategias de enfrentamiento}

Ya hemos visto algunos de los efectos del acoso escolar, abordamos ahora los efectos del estigma, en general, especialmente en aquellos casos en los que concurren varios estigmas, potenciándolos. Tratamos el estigma de género como un estresor a partir de cómo afecta la existencia del estigma a individuos que no han sido estigmatizados explícitamente, pero podrían ser receptores del estigma. Estas consideraciones nos permitirán perfilar algunas de las respuestas dadas por los estigmatizados a los retos de la estigmatización, lo que se conoce como estrategias de coping y empowerment.

La literatura sociológica y psicológica sobre el estigma (Corrigan et al., 2001; Crosby, Bromely y Saxe, 1980; Hebl, Tickel y Heatherton, 2000; Morrison y Von Glinow, 1990) se ha centrado en identificar su impacto, mostrando que los individuos estigmatizados son menospreciados y denigrados por la sociedad y tienen que enfrentarse a numerosas dificultades "adicionales" en su vida diaria: se encuentran más a menudo en interacciones sociales incómodas y equívocas, reciben menos ayuda del entorno social e incluso familiar, pueden tener más dificultades para acceder a un hogar o encontrar un trabajo y, en éste, enfrentan diversos "techos de cristal”. Es una obviedad que todo ello afecta en general a su autoestima y sentimiento de auto-eficacia.

En particular, la estigmatización de gays, lesbianas, bisexuales y transexuales es un fenómeno cultural omnipresente que va desde las formas institucionalizadas de exclusión de derechos -negación del matrimonio o reconocimiento de unión civil, 
adopción o tributación conjunta en la mayoría de los estados del mundo ${ }^{8}$-, hasta formas manifiestas de violencia. Todo ello refuerza la creencia de que las minorías sexuales son menos dignas de derechos y protección que los heterosexuales y crean un entorno hostil para las minorías sexuales. Kertzner et al. (2009) han mostrado que la población LGTB presenta un nivel de morbosidad psiquiátrica más elevado que la población heterosexual (Cochran, Sullivan y Mays, 2003). Las vivencias de discriminación de dichos sujetos acaban causando estrés, locus de control externo, baja autoestima e incremento del malestar emocional y el sentimiento de aislamiento social, haciéndolas más vulnerables a problemas de salud, sobre todo, a enfermedades cardiovasculares, depresión, ansiedad, uso de sustancias, conductas sexuales de riesgo, entre otras (Meyer, 2003). La razón es que el estigma va asociado o se manifiesta como un complejo de estresores, como el prejuicio, la discriminación y la violencia, que constituyen un minority stress al que están sometidos los miembros de los colectivos LGTB considerados como grupo minoritario en la literatura (Meyer, 1995; 2003).

La consideración del estigma como estresor es clara en el estudio de los efectos del estigma no visible. Goffman (1963) distingue, dependiendo de si el estigma es visible o no, entre individuos "desacreditados" (con estigma visible, evidente para los otros) y "desacreditables" (no visible). Se podría pensar que las personas con estigma oculto, como la homosexualidad, evitan el prejuicio y la discriminación que tienen que enfrentar aquellas con uno visible porque pueden esconderlo (Jones et al. 1984). Sin embargo, Pachankis (2007) ha mostrado que las personas con estigma no visible (una orientación sexual no heterosexual, infertilidad, traumas como la violación o estar desempleado) tienen que enfrentarse a un estrés considerable en su vida diaria. Para las personasl LGTB son fuentes de estrés decidir si, cuándo, cómo y a quién revelar el estigma oculto (Greene et al., 2003); la ansiedad de ser descubiertos o estar aislado de quienes comparten la misma condición. Así, esconder un secreto vergonzoso, como un estigma no visible, puede originar una verdadera tensión emocional (Kelly, 2002).

Estas vivencias, además, pueden tener efectos acumulativos. Así, el estigma disuade a muchos individuos con problemas de salud de acudir a los servicios de salud, dificultando la recuperación y aumentando las probabilidades de recaída (Warner et al. 1989; Satcher, 1999; Penn Kohlmaier y Corrigan, 2000). Si, en general, el minority stress afecta a cualquier grupo, dentro de la población LGTB algunos colectivos pueden resultar más afectados cuando varios factores susceptibles de ser objeto de discriminación se cruzan interactuando entre ellos, esto es: las

${ }^{8}$ Solo cinco estados reconocen todos los derechos -Argentina, Dinamarca, España y Holanda, habiendo sido todos ellos de adquisición reciente en la últimas década, incluso hace pocos años, y en algunos estados están siendo parcialmente reconocidos en la actualidad (Alemania, Australia, Brasil, México, Estados Unidos y Uruguay, entre otros). 
condiciones sindémicas (Herrick, Lim, Wei, 2011) ${ }^{9}$, específicamente, la etnia, la edad, el género y la identidad sexual se configuran como factores específicos de estrés. Numerosas investigaciones dan cuenta de estas interacciones: 1) el sexismo y heterosexismo aumenta los niveles de estrés psicológico de mujeres lesbianas y bisexuales (Szymanski, 2005); 2) el rechazo y la falta de integración en las comunidades LGTB por razones de etnia de individuos de origen africano o latino (Me-yer, Schwartz y Frost, 2008); 3) el aumento de la exposición a la discriminación y victimización de los jóvenes que ahora pueden revelar su identidad sexual a más temprana edad. a su familia y amigos, antes que las anteriores generaciones (Floyd y Blakemann, 2006), así como su mayor exposición a la victimización y discriminación por su visibilidad (Braveman, Barclay, 2009; Weich, Patterson, Shaw, Stewart-Brown, 2009); 4) el heterosexismo y las actitudes hostiles que enfrenta la población bisexual por su concreta condición (Ochs, 1996), incluso dentro del ambiente $\mathrm{LGTB}^{10}$. Estos efectos múltiples de discriminación se refuerzan mutuamente y explican la mayor prevalencia de sujetos bisexuales con depresión, ansiedad, intentos de suicidio o abuso de alcohol (Jorm et al., 2002).

El estudio de la sindemia asociada a la estigmatización continúa las líneas tradicionales de estudio de la psicología social. Empero, es necesario recordar que muchas víctimas de la estigmatización son capaces de vivir plena y satisfactoriamente (Miller y Kaiser, 2001) y no todas las personas estigmatizas desarrollan condiciones sindémicas. Algunos estudios muestran cómo un elevado porcentaje de hombres gays (el 77\%) ha sido capaz de resistir al estigma y, a pesar de poder haber desarrollado alguna problemática psicosocial, evitó que otras problemáticas se presentasen conjuntamente (Gwads et al., 2006; Herrick, Lim, Wei, 2011). Esta paradoja se entiende mejor cuando contemplamos el estigma de género como estresor, lo que nos permite profundizar en el conocimiento de las formas en las que las y los estigmatizados manejan el estrés y consiguen tener vidas plenas y satisfactorias a pesar de su estatus (Miller y Major, 2000). Así, es posible analizar los problemas asociados al estigma desde otra perspectiva y ya contamos con investigaciones, si bien todavía minoritarias, sobre los procesos, medios o estrategias que

${ }^{9}$ De forma extremadamente sintética, la sindemia designa un conjunto de problemas de salud mutuamente potenciados en contextos sociales y físicos nocivos, que puede afectar significativamente a la carga de la enfermedad y el nivel de salud de una población (Singer, 2009).

${ }^{10}$ Esta "bifobia” tiene sus raíces en una visión "monosexista” por la cual la sexualidad tiene que dirigirse hacia un género u otro (Rust, 2000), que niega que la bisexualidad como posibilida de práctoca sexo-afectiva legítima o la considera una traición a las identidades gay y lesbiana (Herek, 2002). De hecho, los sujetos bisexuales tienen menos modelos disponibles - un contacto menor con personas de su mismo grupo-, y menor acceso a información concreta y apropiada sobre su situación (Rust, 2002). 
ayudan estas personas a manejar y superar los estigmas y sus efectos y la resiliencia $^{11}$.

La resiliencia no es una idea nueva en psicología. Se ha estudiado, por ejemplo, en adolescentes que consiguen superar las adversidades de un entorno desfavorable (pobreza, estigmas y traumas), que parecían relacionarles con la delincuencia, la enfermedad mental o comportamientos antisociales y cómo tras haber desarrollado competencias y capacidades evitan los resultados negativos a los que parecen abocados, creciendo y viviendo con plenitud (Garmezy, 1991). El estudio de la resiliencia deriva también de una transformación en el paradigma psicológico, el de la "psicología positiva" que no pretende estudiar y curar las consideradas enfermedades mentales sino estudiar los mecanismos para hacer la vida más plena (Seligman y Csikszentmihalyi, 2000). Participando de este cambio de paradigma, se ha propuesto (Kertzner et al., 2009; Keyes, 1998) considerar el bienestar psicológico de las personas no sólo en términos de morbosidad psiquiátrica, sino también de bienestar social. Concepto éste que hace referencia al sentimiento de las personas de pertenecer y contribuir positivamente a la sociedad, de que el mundo tiene sentido y es comprensible, una actitud positiva hacia los demás y la creencia de que la sociedad se desarrollará positivamente. La novedad de estos desarrollos teóricos hace que no haya todavía una terminología claramente establecida ni sea posible el consenso científico en torno a todos los fenómenos estudiados.

La respuesta de estrés se presenta cuando las demandas que el elemento estresante hace al individuo son similares o superan a las capacidades y recursos de la persona para enfrentarse a ellas (Lazarus y Folkman, 1984). De esta forma, el estrés implica una valoración cognitiva respecto a las demandas y a los recursos que la persona piensa tener disponibles para afrontar sus retos. Una respuesta de estrés se presenta cuando el individuo percibe una amenaza que duda pueda superar con facilidad. Así, eventos vinculados al estigma pueden ser percibidos como no estresantes si la persona posee recursos adaptativos que le permitan manejar el potencial estresor. Estos recursos adaptativos pueden ser psicológicos o sociales, conductuales o económicos (Clark et al, 1999; Miller et al., 1995; Miller y Rudiger, 1997). Por esto hay una gran variabilidad en las estrategias de afrontamiento del estigma debido a condiciones individuales, a factores externos - como apoyo del entorno familiar o social-; una variedad que aumenta por la heterogeneidad del colectivo LGTB y, por tanto, por las tipologías del estigma (Masten et al. 1999).

${ }^{11}$ La resiliencia es la capacidad de superar los eventos adversos y ser capaz de tener un desarrollo exitoso a pesar de circunstancias adversas. De latín resilio (Kotliarenco, Cáceres Fontecilla, 1997), que significa volver atrás, volver de un salto o rebotar, implica competencia para afrontar de manera positiva el riesgo o a la adversidad (Luthar, Cushing 1999). Para que aparezca la resiliencia tienen que darse tanto factores de riesgo como de protección que ayuden a conseguir un resultado positivo o reduzcan o eviten un resultado negativo (Becoña, 2006). 
Es importante destacar que las mismas estrategias frente al estigma de género pueden ser entendidas y catalogadas de dos formas diferentes (Oyserman y Swim, 2001; Shih, 2004). Si la estrategia adoptada es reactiva y preventiva se considera como coping, pues sirve para evitar las consecuencias negativas, pero no para crear resultados positivos. Las estrategias proactivas usadas para crear situaciones positivas de crecimiento se consideran empoderamiento (Oyserman y Swim, 2001). En estas estrategias, enfrentarse a las adversidades no implica un desgaste de energías y recursos, sino que crea procesos enriquecedores y regeneradores, que permiten desarrollar sentimientos de control y poder del sujeto sobre su propia vida.

Los análisis sobre los factores de riesgo, las estrategias de enfrentamiento y las capacidades de resiliencia más características de la población LGTB, han identificado tres estrategias psicológicas que pueden ser utilizadas de forma conjunta dependiendo de los casos y la situación del individuo para evitar o superar las consecuencias del estigma (Shih, 2004): a) la compensación; b) las interpretaciones estratégicas del entorno social, y c) la creación de múltiples identidades. En la primera de ellas, la compensación implica que las personas se esfuerzan en ser más perseverantes, asertivas, simpáticas y agradables (Dion y Stein, 1978, Miller et al. 1995), refinando sus habilidades sociales, monitoreándolas con más cuidado o accediendo a adoptar el punto de vista de sus interlocutores, revirtiendo su estatus devaluado y aumentando su competencia social (Miller y Myers, 1998).

En la segunda estrategia, las interpretaciones estratégicas del entorno social, las personas manipulan estratégicamente la interpretación de su entorno social para proteger su autoestima. Por ejemplo, en situaciones difíciles pueden convencerse de que han actuado mejor que otros miembros de su grupo, lo que aumenta su sentimiento de auto-eficacia (Crocker y Major, 1989; Crocker y Quinn, 2000). Otra manifestación de este mecanismo sería atribuir el hecho o fracaso a la pertenencia a un grupo estigmatizado y no tanto a sí mismo, protegiendo su autoestima al atribuir a otros la responsabilidad de ciertos resultados negativos. Moghaddam et al. (1995) han demostrado que, por ejemplo, las personas blancas tienden a atribuirse a sí mismas el fracaso en mayor medida que las negras, quienes lo atribuyen más a la discriminación racial. De forma parecida, las personas estigmatizadas pueden negar o minimizar la discriminación que sufren, afirmando que perciben un mayor nivel de prejuicio y discriminación hacia su grupo más que hacia sí mismos (Kessler, Mummendey y Lessie, 2000).

La última de las estrategias se refiere a la capacidad de recurrir a identidades alternativas para protegerse del estigma. Aunque tendamos a concentrarnos en una sola identidad, los individuos son portadores de múltiples identidades (Hewstone, 2000). Así, aunque las personas estigmatizadas tienden a ser definidas por su identidad estigmatizada, pueden ser definidas por otras, como la profesion, nivel de estudios, religión o aficiones. Dado que los estigmas son construcciones sociales, determinadas identidades son estigmatizadas en algunos contextos pero no en otros, por lo que las personas pueden estratégicamente enfatizar determinadas identidades según el contexto social en el que se encuentren, eligiendo la más apta a la situación. Esta idea enfatiza la potencialidad de la agencia humana. Por ejemplo, se ha demos- 
trado que poseer y jugar con múltiples identidades permite protegerse y sentirse más a gusto en diferentes contextos. Ello hace a las personas con personalidades polifacéticas más resistentes al estrés y a la depresión (Linville, 1987) y más satisfechas con su vida (Thoits, 1986).

A continuación sintetizamos a través de algunas situaciones concretas propias de la población LGTB cómo múltiples factores se entrecruzan, influyendo en la elección de las estrategias de coping y de empowerment para afrontar el estigma. Balsam y Mohr (2007), siguiendo a Miller y Kaiser (2001) han enumerado algunas de las estrategias de coping/empowerment frente a los estigmas vinculados a orientaciones sexuales no normativas.

1) Una estrategia de empowerment es la identificación con y participación en una comunidad (D’Augelli y Garnets, 1995). Las comunidades LGTB proveen de recursos, socialización y aceptación a sus miembros (Harper y Scheneider, 2003). La implicación en una comunidad puede ser particularmente relevante como amortiguador frente el impacto de la discriminación, hasta el punto de ser considerada uno de los más importantes factores de resiliencia. Meyer (2003) subraya que el sentido personal de conexión con una comunidad puede mejorar los efectos adversos del estigma empujando los miembros de la comunidad a compararse con otros LGTB más que con los heterosexuales.

2) Otra estrategia de afrontamiento del estigma es la revelación de la propia identidad sexual (outness level). Se trata de una estrategia controvertida que muestra los costes del estigma, pues al desarrollo de una identidad positiva como miembro de un grupo oprimido (Reynolds y Hanjorgiris, 2000) se puede contraponer la estrategia del passing (hacerse pasar por) que se utiliza cuando una estigma y quien lo porta no son completamente visibles (Snorton, $2009)^{12}$. Las personas LGTB pueden vivir un dilema respecto a cómo presentarse a los demás: si salen del armario, se arriesgan a ser discriminados, perder su trabajo, amigos o familia por lo que pueden esconder la homosexualidad como una estrategia consciente para evitar consecuencias negativas, después de un análisis de sus costes y beneficios (Kelly 2002). Pero esta es una opción problemática, ya que mantener el secreto puede fomentar un sentimiento de hipocresía y distancia en las relaciones, pues las personas que guardan secretos personales tienden a ser más solitarias, tímidas, introverti-

${ }^{12}$ Un transexual MTF que se ha sometido a una intervención quirúrgica para convertirse en mujer puede decidir hacerse pasar por mujer sin revelar su condición de transexual. De todos modos Kando (1972) cuenta cómo los transexuales presentan una peculiar condición, afirmando que el transexual será considerado mujer en los nuevos contextos, mientras será considerado hombre en los contextos donde ya era conocido. 
das y ansiosas (Cepeda-Benido y Short, 1998) ${ }^{13}$. Además, el ocultamiento se considera un síntoma de falta de adaptación, vergüenza o sentimiento de culpa, pues los secretos son vistos como falta de confianza hacia los demás y hacia sí mismos y el simple hecho de tener que esconder el estigma puede hacer creer a quien lo guarda que lo escondido es, en sí, vergonzoso, por ser motivo de ocultación. Paradójicamente, la normalización de la homosexualidad manifiesta ha llevado a la patologización de la homosexualidad oculta (Cain, 1989), como si la decisión de revelar o no la orientación sexual equivaliese al nivel de aceptación de la persona.

Una de las líneas de estudio (McCarn y Fassinger, 1996) de este problema mantiene que el grado de revelación sería reflejo del grado de aceptación por el contexto social de la visibilidad de la identidad LGTB. El outness level estaría, así, más vinculado a factores socio-contextuales - de apoyo social-, que a factores de adaptación psicológica. Otros estudios mantienen que salir del armario es signo de la formación de una identidad positiva y está asociado o puede llevar a una mayor auto-aceptación (Cass, 1979). Así, gays y lesbianas que revelan el propio estatus a los padres muestran mayor autoestima (Gershon, Tschann y Jemerin, 1999; SavinWilliams, 1999).

Herek (2003) considera el dilema desde un punto de vista distinto, relacionado con el empowerment. La revelación de una identidad no heterosexual puede aportar beneficios porque los eventuales feedback harían crecer la confianza de la persona y favorecer su (auto) aceptación y el desarrollo de una identidad positiva. Este caso muestra los efectos positivos de las estrategias de empowermment, frente a las del mero coping.

Los grupos LGTB o minorías sexuales y sus comunidades han mostrado una fuerza considerable en los últimos 40 años frente a manifestaciones a menudo extremas de homofobia y marginalización. Han asumido y revertido los valores de los términos e imágenes negativos con los que se les denigraba -pedé, marica, Schwul, bollera, camionera, marimacho, maricón, travelo, etcétera. Aún más, las comunidades han utilizado las adversidades (por ejemplo, la epidemia de HIV) como una fuerza motivadora para organizarse y exigir derechos e igualdad en todos los aspectos de la vida. Esta resiliencia colectiva ha ayudado a las comunidades a superar retos importantes y a crecer con fuerza. Sería importante desarrollar una teoría de la resiliencia para disponer de un modelo que permitiera aprovechar de

${ }^{13}$ Frable et al. (1998) mostraron que los estudiantes que escondían rasgos estigmatizables mostraban menos confianza social, mayor ansiedad y depresión, y autoestima más baja que los estudiantes con un estigma visible o sin estigma. En un estudio, los hombres gays que escondían su estigma mostraban más depresión y menos bienestar general psicológico frente a los que lo habían revelado (Ullrich, Lutgendorf and Stapleton, 2003). 
esta fuerza e incrementar los resultados de los esfuerzos para promover la salud de la población LGTB (Herrick, Lim y Wei, 2011).

\section{Algunas consideraciones finales}

Para designar la estigmatización por la ruptura de los modelos heteronormativos, Herek acuñó el término "estigma sexual”. Sin embargo, este término corre el riesgo de naturalizar el estigma al fijarlo en la sexualidad como hecho biológico y es impreciso, pues el estigma pretende controlar no solo los sentimientos y prácticas privadas sino que sanciona conductas y modelos más amplios y públicos: el género y su performance. El concepto de estigma de género permite entonces analizar conjuntamente los procesos de estigmatización de las mujeres y de las personas LGTB.

Dado que el proceso de aprendizaje de las normas de género tiene un claro componente hedónico, el nuevo modelo del condicionamiento valorativo puede aportar información valiosa sobre los procesos de transmisión aprendizaje de estas normas e incrementar la libertad en la elección de los individuos al eliminar coacciones y violencias, especialmente en el sistema escolar.

El bullying o acoso escolar de género no es sólo una manifestación del estigma, sino uno de los procedimientos fundamentales de su transmisión-aprendizaje, por lo que la comunidad escolar ha de crear ambientes escolares que lo impidan, por ejemplo, fomentando grupos de pares inclusivos e igualitarios, pues los grupos jerarquizados precisan de la distinción del "nosotros" vs. "ellos", fomentando la estigmatización y la violencia. Pero la escuela no solo ha de adoptar un papel reactivo sino que como parte de una enseñanza compensadora de las desigualdades, ha de ser, en sí misma, un modelo de convivencia en el respeto y ha de proporcionar modelos que reflejen la diversidad y la igualdad, y garanticen la convivencia y el respeto a la diferencia, incluyendo la diversidad sexo-afectiva y de género. Uno de los objetivos principales del sistema educativo es el empoderamiento de los estudiantes, que solo se consigue a través del fomento de la autonomía. En el caso de la población LGTB es posible imaginar que los programas escolares que ofertan modelos y espacios de empowerment como las Gay-Straight Alliance ${ }^{\text {s14, }}$ pueden ayudar los jóvenes a empoderarse y arreglárselas con el bullying de género.

${ }^{14}$ La Gay-Straight Alliance (Alianza Gay-Heterosexual - GSA) son clubes estudiantiles autogestionados ubicados en escuelas secundarias que trabajan contra la homofobia y proporcionan un lugar seguro para el encuentro, apoyo mutuo, comunicación y reflexión sobre cuestiones relacionadas con la orientación sexual. Algunas GSAs trabajan en la autoeducación de los miembros y del conjunto de la comunidad escolar sobre la orientación e identidad sexual. Entre sus actividades se cuentan la organización de conferencias sobre el mundo LGTB, de la "Semana del Orgullo", talleres educativos, paneles y otras celebraciones. Muchas participan en el Día de Silencio, en el que los participantes permanecen calla- 
La acuñación del concepto de homofobia, que revertía la culpa sobre los que odiaban a aquellos no conformes con la norma de género; la adopción y reversión del valor connotativo de los conceptos despectivos que designaban a los diferentes miembros de las minorías de género o la movilización frente al VIH son claros ejemplos de estrategias socio-políticas de empoderamiento del conjunto de la comunidad LGTB. Saber de qué fuerzas disponen las poblaciones estigmatizadas y cómo estos elementos pueden usarse para desarrollar resiliencia, es difícil. La resiliencia ha recibido poca atención pero parece revelar un gran potencial para promover la salud dentro del colectivo LGTB y "en general” para todos los seres humanos. Además de continuar tratando de eliminar los estigmas, y en consideración a que este es un proceso de larga duración, se deberían mejorar las vidas cotidianas de los grupos estigmatizados. La investigación podría identificar qué factores permiten a los individuos enfrentarse y sobreponerse a los estigmas para vivir su vida de forma plena y satisfactoria.

\section{Nota:}

Esta publicación ha sido producida con el financiamiento de HERMES European Project (2011-2013) del Daphne III Programme, Comisión Europea. El contenido de esta publicación es de sola responsabilidad del autor y no refleja necesariamente la posición de la Comisión Europea.

dos durante todo el día para de demostrar el silencio impuesto por la homofobia. Algunas GSAs organizan el día de "Enseñar a los Maestros" con el personal educativo para enseñarles a ser mejores aliados de estudiantes LGTB. Otras GSAs son clubes activos y trabajan para conseguir que las cuestiones de los LGTB estén representadas en el plan de estudios, compra de libros relacionados para la biblioteca e implementación de políticas antidiscriminación a nivel de distrito. Las GSAs son un modo de construir una comunidad escolar que disminuya el aislamiento al que, de otra manera, los estudiantes LGTB estarían expuestos. Véase en Internet: http://www.gsanetwork.org/ 


\section{Bibliografía}

Balsam, F. B., Mohr, J. J. (2007): "Adaptation to Sexual Orientation Stigma: A Comparison of Bisexual and Lesbian/Gay Adults". Journal of Counselling Psychology 54, 3, 306-319.

Barón V., S. y Lacalle, rosa (2011): Teatro y TICs al Servicio de la Prevención del Acoso Escolar, Presentación del Proyecto del IES Manuel Patarroyo de Parla. Madrid, en Internet: http://ies.manuelelkinpatarroyo.parla.educa.madrid.org/

Bacchini, D. (2000) Il bullismo nelle varie età, Roma: Babele

Barret, B. and Logan, C. (2002): Counseling gay men and lesbians: A practice primer. Pacific Grove, CA: Brooks/Cole Publishing Company.

Bauman, Z. (2005): Identidad. Madrid: Losada.

Becoña, E. (2006): "Resiliencia: definición, características y utilidad del concepto". Revista de Psicopatología y Psicología Clínica 11, 3, 125-146.

Bieke, D. (2011) "The effect of disgust conditioning and disgust sensitivity on appraisals of moral transgressions". Personality and individual differences 50, 7, 1142-1146.

Braveman, P., Barclay, C. (2009): "Health disparities beginning in childhood: a lifecourse perspective". Pediatrics 124, 3, 163-75.

Butler, J, (1990): Gender Trouble: Feminism and the Subversion of Identity. New York and London: Routledge.

Butler, J, (1993): Bodies that Matter. On the Discursive Limits of "Sex". New York and London: Routledge.

Cain, R. (1991): "Stigma management and gay identity development". Social Work 36, 67-73.

Cass, V. C. (1979): "Homosexual identity formation: A theoretical model". Journal of Homosexuality 4, 219-235.

Cepeda- Benido, A., Short, P. (1998): "Self-concealment, avoidance of psychological services, and perceived likelihood of seeking professional help". Journal of Counseling Psychology 45, 58-64.

Chodorow, N. J. (1994): Femininities, Masculinities, Sexualities: Freud and Beyond. Lexington : The University Press of Kentucky.

Clark, R., Anderson, N. B., Clark, V. R., Williams, D. R. (1999): "Racism a stressor for African Americans: a bio psychological model". American Psychologist 54, 805-816.

Clarke, V.; Kitzinger, C. and Potter, J. (2004): "Kids are just cruel anyway: lesbian and gay parents talk about homophobic bullying". British Journal of Social Psychology 43, 4, 531-550.

Cochran, S. D., Sullivan, G. J., Mays, V. M. (2003): "Prevalence of mental disorders, psychological distress, and mental health service use among lesbian, gay and bisexual adults in the United States". Journal of Consulting and Clinical Psychology 71, 53-61.

Coleman E. (1982): "Developmental stages of coming-out process". American Behavioral Scientist 25, 469-482. 
Cope, A., Darke, J. (1999): Trans Accessibility Project: Transphobia And Discrimination, from http://www.queensu.ca/humanrights/2main.htm

Corrigan, P. W., Green, A., Lundin, R., Kubiak, M. A., Penn, D. L. (2001): "Familiarity with and social distance from people who have serious mental illness". Psychiatric Services 52, 953-58.

Crocker, J., Major, B. (1989): "Social stigma and self-esteem: the self-protective properties of stigma". Psychological Review 96, 608-30.

Crocker, J., Major, B., Steele, C. (1998): "Social Stigma", en Gilbert, DT, Fiske, ST. (eds.): The Handbook of Social Psychology (504-53). Boston, MA: McGraw Hill.

Crocker, J., Quinn, D. M. (2000): "Social stigma and the self: meaning, situations and self-esteem" In Heartherton, T. F.; Kleck, R. E.; Hebl, M. R. and Hull, J. G. (eds.): The social psychology of stigma (153-83): New York: Guilford.

Crosby, R., Bromely, S., Saxe, L. (1980): "Recent unobtrusive studies of black and white discrimination and prejudice: a literature review". Psychological Bulletin 87, 546-63.

D’Augelli, A. R., and Garnets, L. D. (1995): "Lesbian, gay, and bisexual communities", en D'Augelli, A. R. and Patterson, C. J. (eds.): Lesbian, gay and bisexual identities over the lifespan: Psychological perspectives ( 293-320): New York: Oxford University Press.

De Houwer, J., Thomas, S., and Baeyens, F. (2001): "Associative learning of likes and dislikes: A review of 25 years of research on human evaluative conditioning". Psychological Bulletin 127, 853-869.

De Houwer, J. (2007): "A Conceptual and Theoretical Analysis of Evaluative Conditioning". The Spanish Journal of Psychology 10, 2, 230-241.

Dion, K. K., Stein, S. (1978): "Physical attractiveness and interpersonal influence". Journal of Experimental Social Psychology 14, 97-108.

Doise, W., Deschamps, J.-C., and Mugny, G. (1980): Psicologia social experimental. Barcelona: Hispano Europea.

Espelage, D. L.; Aragon, S. R. and Birkett, M. (2008): "Homophobic Teasing, Psychological Outcomes, and Sexual Orientation Among High School Students: What Influence Do Parents and Schools Have?". School Psychology Review 37, 2, 202-216.

Floyd, F. J., Blakemann, R. (2006): "Coming-out across the life course: implication of age and historical context". Archives of Sexual Behavior 35, 287-296.

Fonzi, A. (1997): Il bullismo in Italia. Firenze: Giunti.

Frable, D. E. S., Platt, L., Hoey, S. (1998): "Concealable stigmas and positive selfperceptions: feeling better around similar others". Journal of Personality and Social Psychology 74, 909-922.

Garmezy, N. (1991): "Resilience and vulnerability to adverse developmental outcomes associated with poverty". American Behavioral Scientist 34, 416-430.

Generelo, J.; Pichardo, J. I. (2005) Homofobia en el sistema educativo, Madrid: COGAM

Generelo, J. (2012) Acoso escolar homofóbico y riesgo de suicido en adolescentes y jóvenes. Área de Educación de FELGTB - Comisión de Educación de COGAM, 
http://www.felgtb.org/rs/1584/d112d6ad-54ec-438b-93584483f9e98868/91c/filename.

Gershon, T. D., Tschann, J. M., and Jemerin, J. M. (1999): "Stigmatization, selfesteem, and coping among the adolescent children of lesbian mothers". Journal of Adolescent Health 24, 437-445.

Goffman, E. (1963): Stigma: Notes on the management of Spoiled Identity, New York: Simon \& Schuster Inc.

Greene, K., Derlega. V. J., Yep, G. A., Perronio. S. (2003): Privacy and disclosure of HIV in interpersonal relationships: a sourcebook for researchers and practitioners. Mahwah, NJ: Erlbaum.

Gwadz, M. V., Clatts, M. C., Huso Yi, Leoanar, N. R. (2006): "Resilience among young men who sex with men in New York City". Sexuality Research and Social Policy: Journal of NSRC 3, 1, 13-21.

Haaga, D.A.F. (1991): "Homophobia?". Journal of Social Behavior and Personality 6, 171-174.

Hall, S. (2003): Identidad y globalización. Cuestiones de identidad cultural. Buenos Aires: Amorrortu Editores.

Harper, G. W. y Schneider, M. (2003): "Oppression and discrimination among lesbian, gay, bisexual, and transgendered people and communities: A challenge for community psychology". American Journal of Community Psychology 31, $243-252$.

Harris, J. (1999): El mito de la educación. Por qué los padres pueden influir muy poco en sus hijos, Barcelona: Grijalbo.

Hebl, M. R., Tickel, J., Heatherton, T. F. (2000): "Awkward moments in interactions between nonstigmatized and stigmatized individuals", en Heartherton,T. F.; Kleck, R. E.;Hebl, M. R. and Hull, J. G. (eds.): The social psychology of stigma (275-306). New York: Guilford

Herek G. M. (1984): "Beyond "homophobia": A social psychological perspective on attitudes towards lesbians and gay men". Journal of Homosexuality 10, 1-2, $1-21$.

Herek G. M. (1986): "On heterosexual masculinity: some psychical consequences of the social construction of gender and sexuality", American Behavioral Science 29, 563-77.

Herek G. M. (1996): "Heterosexism and homophobia", en Cabaj, R. P.; Stein T. S. (eds.): Textbook of homosexuality and mental health, 101-113. Washington, DC: American Psychiatric Press.

Herek G. M. (2002): "Heterosexuals' attitudes toward bisexual men and women in the United States". Journal of Sex Research 39, 264-274.

Herek G. M. (2003): "Why tell if you're not asked? Self-disclosure, intergroup contact, and heterosexuals' attitudes toward lesbians and gay men", en Garnets, L. D. and Kimmel, D. C. (eds.): Psychological perspectives on lesbian, gay, and bisexual experiences, 270-298, New York: Columbia University Press. 
Herek G. M. (2004) "Beyond "homophobia": thinking about sexual prejudice and stigma in the Twenty-First Century". Sexuality Research \& Social Policy Journal of NSRC1: 2, 6- 24.

Herek G. M. (2007): "Confronting sexual stigma and prejudice: theory and practice", Journal of Social Issues 63, 905-925.

Herek G. M. (2008): "Sexual Prejudice", en Nelson, T. (ed.): Handbook of prejudice (439-465). Mahwah, NJ: Erlbaum.

Herek, G.M., Berrik, K. T. (eds). (1992): Hate Crimes: confronting violence against lesbians and gay men. Thousand Oaks: Sage.

Herek, G.M., Chopp, R., Strohl, D. (2007): "Sexual Stigma: putting sexual minority health issues in context", en Meyer, I. and Northridge, M. (eds): The health of sexual minorities: Public health perspectives on lesbian, gay, bisexual and transgender population (171-208). New York: Springer.

Herek, G. M., Cogan, J. C., Gillis, J. R., and Glunt, E. K. (1998): "Correlates of internalized homophobia in a community sample of lesbians and gay men". Journal of the Gay and Lesbian Medical Association 2, 17-25.

Herek, G.M., Gillis, J. R., Cogan, J. C. (2009): "Internalized stigma among sexual minority adults: Insights from a social psychological perspective", Journal of Counseling Psychology 56, 1, 32-43.

Herek, G. M. and Glunt, E. K. (1995): "Identity and community among gay and bisexual men in the AIDS era", en Herek, G. M. and Greene, B. (eds.): AIDS, identity, and community: The HIV epidemic and lesbians and gay men, 55-84, Thousand Oaks, CA: Sage.

Herrick A. L., Lim, S. H., Wei, C. (2011): "Resilience as un Untapped Resource in Behavioral Intervention Design for Gay Men", AIDS Behav 15, 25-29.

Hewstone, M. (2000): "Contact and categorization: social psychological interventions to change intergroup relations", en Stangor, C. (ed.): Stereotypes and prejudice: essential readings. 394-418, Philadelphia: Taylor and Francis.

Irigaray, L. (1977): Ce sexe qui n'en est pas un. Paris: Editions de Minuit.

Izzo, M. (2007): Tra(n)scritti politici 2000-2007 (My Political Testament), Genova: Mirella Izzo.

Jones, E., Farina, A., Hastorf, A., Markus, H., Miller DT, Scott, R. (1984): Social Stigma: The Psychology of Marked Relationshipsm, New York: Freeman.

Jorm, A. F., Korten, A. E., Rodgers, B., Jacomb, P. A., Chirstensen, H., (2002): "Sexual orientation and mental health: results from a community survey of young and middle-aged adults", British Journal of Psychiatry 180, 423-427.

Kando, T. (1972): "Passing and stigma management: the case of the Transexual", The Sociological Quartertly 13: 4, 475-483.

Kelly, A. E. (2002): The psychology of secrets, New York: Kluwer Academic/Plenum.

Kertzner, R. M., Meyer, I. H., Frost,D. M., Stirratt, M. J. (2009): "Social and psychological well-being in lesbians, gay men and bisexuals: the effects of race, gender, age and sexual identity", American Journal of Orthopsychiatry 79, 4, 500-510. 
Kessler, T, Mummendey A., Lessie, U. (2000): "The personal-group discrepancy: is there a common information basis for personal and group judgment?". Journal of Personality and Social Psychology 79, 95-109.

Keyes, C. L. M. (1998): "Social well-being", Social Psychology Quarterly 61, 121140.

Kitzinger, C. (1986): "Heteopatriarchal language: The case against "homophobia", Gossip 5, 15-20.

Kotliarenco, M. A., Cáceres, I. Fontecilla, M. (1997): Estado de arte en resiliencia,Washington: Organización Panamericana de la Salud.

Lazarus, R. S., Folkman, S. (1984): Stress, appraisal and coping. New York: Springer.

Lingiardi, V., Luci, M. (2006): "L’omosessualità in psicoanalisi", en P. Rigliano, M. Graglia (a cura di) Gay e lesbiche in psicoterapia, 1-72, Milano: Raffaello Cortina Editore.

Link, B. G., Phelan, J. C. (2001): "Conceptualizing Stigma", Annual Review of Sociology 27, 363-385.

Linville, P. (1987): "Self-complexity a cognitive buffer against stress-related illness and depression", Journal of Personality and Social Psychology 52, 663-76.

Luthar, S. S., Cushing, G. (1999): "Measurement issues in the empirical study of resilience. A critical evaluation and guidelines for future work", Child Development 71, 543-562.

Mason, A.; Palmer, A. (1996): Queer Bashing: A National Survey of Hate Crimes Against Lesbians and Gay Men, London: Stonewall.

Masten, A. S, J. J. Hubbard, S. D. Gest, A. Tellegen, N., Garmezy, and M. Ramirez. (1999): "Competence in the context of adversity: Pathways to resilience and maladaptation from childhood to late adolescence", Development and Psychopathology 11,143-69.

McCarn, S. R., and Fassinger, R. E. (1996): "Revisioning sexual minority identity formation: A new model of lesbian identity and its implications", Counseling Psychologist 24, 508-534.

Merckelbach, H, de Jong, P., Arntz, A, (1993): "The role of evaluative learning and disgust sensitivity in the etiology and treatment of spider phobia", Advances in Behaviour Research and Therapy 15, 4, 243-255.

Meyer, I. H. (1995): "Minority stress and mental health in gay men", Journal of Health and Social Behavior 36, 339-367.

Meyer, I. H. (2003): "Prejudice, social stress, and mental health in lesbian, gay and bisexual populations: conceptual issues and research evidence", Psychological Bulletin 129, 674-697.

Meyer, I. H., Schwartz, S., Frost, D. M. (2008): "Social patterning of stress and coping. Does disadvantaged social status confer more stress and fewer coping resources?", Social Science \& Medicine, doi:10.1016/j.socscimed.2008.03.012

Miller, C. T., Rothblum, E. D. Felicio, D., Brand P. (1995): "Compensating for stigma: obese and nonobese women's reactions to being visible", Personality and Social Psychology Bulletin 21, 1093-1106. 
Miller, C. T. y Rudiger, L. (1997): Compensation for prejudice: how the stigmatized obtain desired outcomes despite prejudice; Paper presented at the annual meeting of the Society of Experimental Social Psychology, Toronto, Ontario, Canada.

Miller, C. T. y Major, B. (1998): "Compensating for prejudice: how obese people (and others) control outcomes despite prejudice", en Swim, J. K. and Stangor, C. (eds.). Prejudice: The target's perspective, 191-218, San Diego, CA: Academic Press.

Miller, C. T. y Major, B. (2000): "Coping with stigma and prejudice", en Heathertron, T. F. and Kleck, R. E. (eds). Social psychology of stigma, 243-272, New York: Guilford Press.

Miller, C. T., Kaiser, C. R. (2001): "A theoretical perspective on coping with stigma", Journal of Social Issues 57, 1, 73-92.

Moghaddam, F. M., Taylor, D. M., Lambert, W. E., Schmidt, A.E. (1995): "Attributions and discriminations: a study of attributions to the self, the group and the external factors among whites, blacks and Cubans in Miami", Journal of CrossCultural Psychology 26, 209-20.

Morrison, A. M., Von Glinow, M. (1990): "Women and minorities in management", American Psychologist 45, 200-208.

Murdock, T. B.; Bolch, M. B. (2005): Risk and Protective Factors for Poor School Adjustment in Lesbian, Gay, and Bisexual (LGB) High School Youth: Variable and Person-Centered Analyses, Psychology in the Schools 42, 2,159-172.

Nyathi, (2008): "Reducing prejudice through evaluative conditioning: Use of the self as an unconditioned stimulus in evaluative conditioning" (Dissertationsprojekt).

Norton, J. (1997): "Brain says you're a girl, but I think you're a sissy boy: cultural origins of transphobia". Journal of Gay, Lesbian and Bisexual Identity 2, 139164.

Ochs, R. (1996): "Biphobia: It goes more than two ways", en B. A. Firestein (ed.): Bisexuality: The psychology and politics of an invisible minority ( 240-259). Newbury Park, CA: Sage

Ochs, R., Deihl, M. (1992): "Moving beyond binary thinking", en W. J. Blumenfeld (ed.). Homophobia: how we all pay the price (67-75). Boston: Beacon Press.

Olson, M. and Fazzio, R. (2006): "Reducing automatically activated racial prejudice through implicit evaluative conditioning". Society for Personality and Social Psychology 32, 4, 421-433.

Olweus, D. (1993): Il bullismo a scuola. Florencia: Giunti

Oyserman, D., Swim, J. K. (2001): "Stigma: an insider's view". Journal of Social Issues $57,1,1-14$.

Pachankis, J. E., (2007): "The psychological implications of concealing a stigma: a cognitive-affective-behavioral model". Psychological Bulletin 133, 2, 328-342.

Penn, D. L., Kohlmaier, J. R., Corrigan, P. W. (2000): "Interpersonal factors contributing to stigma of schizophrenia: social skills, perceived attractiveness and symptoms". Schizophrenia research 45, 37-45. 
Pharr, S. (1988): Homophobia: A weapon of sexism. Little Rock, AR: Chardon Press.

Plummer, D. C. (2001): "The quest for modern manhood". Journal of Adolescence $24,15-23$.

Poteat, V. P. (2007): "Peer group socialization of homophobic attitudes and behaviors during adolescence", Child Development 78, 6, 1830-42.

Poteat, V. P.; Espelage, D. L. and Green, H.D. (2007): "The socialization of dominance: peer group contextual effects on homophobic and dominance attitudes", Journal of Personality and Social Psychology 96, 6, 1040-1050.

Prati, G.; Pietrantoni, L. and Norcini, A. (2009): Determinanti del comportamento prosociale in caso di bullismo omofobico, Psicologia della salute 3, 2, 237-254.

Prati, G.; Coppola, M. and Saccà, F. (2010) Report finale della ricerca nazionale sull bullismo omofobico nelle scuole superiore italiane. http://www.arcigay.it. Retrieved 11.12.2012.

Reynolds, A. L., and Hanjorgiris, W. F. (2000): "Coming out: Lesbian, gay, and bisexual identity development", en R. M.. Pérez, K. A. DeBord y K. J. Bieschke (eds.), Handbook of counseling and psychotherapy with lesbian, gay, and bisexual clients, 35-56, Washington, DC: American Psychological Association.

Rivers, I. (2004): "Recollections of bullying at school and their long-term implications for lesbians, gay men, and bisexuals", Crisis 25, 4, 169-75.

Rizzo, D. (2006): "Premessa", en Rizzo, D. Omosapiens Studi e ricerche sull'orientamento omosessuale (xi-xv), Carrocci, Roma, 2006.

Russell, G. M. and Richards, J. A. (2003): "Stressor and resilience factors forlesbians, gay men, and bisexuals confronting antigay politics" Bieschke, American Journal of Community Psychology 31, 313-328.

Rust, P. C. R. (2000): "The biology, psychology, sociology, and sexuality of bisexuality", en Rust, P. C. R. (ed.): Bisexuality in the United States, 403-470, New York: Columbia University Press.

Rust, P. C. R. (2002): "Bisexuality: The state of the union", Annual Review of Sex Research 13, 180-240.

Salmivalli, C. et al. (1996): "Bullying as a group process: Participant roles and their relations to social status within the group", Aggressive Behaviour 22, 1-15.

Satcher, D. (1999): Mental health: a report of the surgeon general; Washington, DC: Department of Health and Human Services.

Savin-Williams, R. C. (1989): "Coming out to parents and self-esteem among gay and lesbian youths", Journal of Homosexuality 18, 1-35.

Sayce, L. (1998): "Stigma, discrimination and social exclusion: What's in a word?", Journal of Mental Health 7, 4, 331-343.

Sedgwick, E. K. (1993): "How to bring your kids up gay", en Warner, M. (ed.) Fear of a queer planet: Queer politics and social theory (69-81), Minneapolis, MN: University of Minnesota Press.

Seger, C. A. (1998): Multiple forms of implicit learning. Sage Publications, Inc.

Seligman, M.E.P. and Csikszentmihalyi, M. (2000): "Positive Psychology: An introduction", American Psychologist 55, 1, 5-14. 
Shih, M. (2004): "Positive Stigma: examining resilience and empowerment in overcoming stigma". Annals of the American Academy of Political and Social Science 591, 175-185.

Sidanius, J. Pratto, F. (1999): Social Dominance, Cambridge: Cambridge University Press.

Singer, M. (2009): Introduction to syndemics - A critical systems approach to public and community health; San Francisco: John Wiley \& Sons.

Smith, P. K. et al. (1999): The nature of school bullying: A cross-national perspective. Florence, KY: Taylor \& Frances/Routledge.

Snorton, R. (2009): "A New Hope": The Psychic Life of Passing". Hypatia 24, 3, 77-92.

Szymanski, D. M. (2005): "Heterosexism and sexism as correlates of psychological distress in lesbians", Journal of Counseling and Development 83, 335-360.

Thoits, P. A. (1986): "Multiple identities: examining gender and marital status differences in distress", American Sociological Review 48, 174-87.

Tobin, D., Menon, M., Menon, M., Spatta, B., Perry, D. G., (2010): "The Intrapsychics of Gender: A Model of Self-Socialization". Psychological Review 117, 2, 601-622.

Ullrich, P. M., Lutgendorf, S. K., Stapleton, J. T. (2003): "Concealment of homosexual identity, social support and CD4 cell count among HIV-seropositive gay men". Journal of Psychosomatic Research 54, 205-212.

Walter, E. (2001): "Guilty by mere association: evaluative conditioning and the spreading attitude effect", Journal of Personality and Social Psychology 82, 6, 919-934.

Warner, M. (1991): "Introduction: Fear of a Queer Planet". Social Text 9, 4, 29, 317

Warner, R., Taylor, D., Powers, M., Hyman, J. (1989): "Acceptance of the mental illness label by psychotic patients: effects on function", American Journal of Orthopsychiatry 59, 398-409.

Weiberg, G. (1972): Society and the Healthy Homosexual, St. Martin’s Press, New York.

Weich, S., Patterson, J., Shaw, R., Stewart-Brown, S. (2009): "Family relationships in childhood and common psychiatric disorders in later life: systematic review of prospective studies", Br. J Psychiatry 194:5, 392-8. 